\title{
Pion electronic decay and lepton universality
}

\author{
Dinko Počanić ${ }^{\star}$, for the PEN Collaboration \\ University of Virginia, Charlottesville, VA 22904-4714, USA \\ ^ pocanic@virginia.edu \\ PAUL SCHERRER INSTITUT \\ Review of Particle Physics at PSI \\ doi:10.21468/SciPostPhysProc.5
}

\begin{abstract}
In common with a number of simple processes involving elementary particles, charged pion decays are profoundly shaped by applicable Standard Model (SM) symmetries and properties. Given the highly precise SM theoretical description, pion decays are used as selective probes of SM parameters, and of possible SM extensions. The PEN experiment at PSI is studying the $\pi^{+} \rightarrow e^{+} v_{e}(\gamma)$, or $\pi_{e 2(\gamma)}$ decay. The primary goal is to reach the relative precision of $5 \times 10^{-4}$ in $R_{e / \mu}^{\pi}$, the branching ratio for $\pi_{e 2(\gamma)}$ decay. We review the PEN research program, its present status, and prospects.
\end{abstract}

(c) (i) Copyright D. Počanić et al.

This work is licensed under the Creative Commons

Attribution 4.0 International License.

Published by the SciPost Foundation.
Received 12-05-2021

Accepted 16-06-2021

Published 06-09-2021

doi:10.21468/SciPostPhysProc.5.025

\subsection{Motivation}

Immediately following the discovery of the charged pion, its decays presented a puzzle: absence of the direct pion decay to an electron that persisted for over a decade. The solution to the puzzle became prominent among the early arguments for the $\boldsymbol{V}-\boldsymbol{A}$ form of the "universal" weak interaction [1]. The $V-A$ helicity suppression of the right-handed state of the electron led to the accurate prediction [2] of the tree level $\pi \rightarrow e \bar{\nu}(\gamma)$, or $\pi_{e 2(\gamma)}{ }^{1}$ branching fraction, $R_{e / \mu, 0}^{\pi}$, even before the decay itself was discovered [3]:

$$
R_{e / \mu, 0}^{\pi} \equiv \frac{\Gamma(\pi \rightarrow e \bar{v})}{\Gamma(\pi \rightarrow \mu \bar{v})}=\frac{m_{e}^{2}}{m_{\mu}^{2}} \cdot \frac{\left(m_{\pi}^{2}-m_{e}^{2}\right)^{2}}{\left(m_{\pi}^{2}-m_{\mu}^{2}\right)^{2}} \simeq 1.283 \times 10^{-4} .
$$

In the years that followed, $\pi_{e 2}$ decay served as an important theory testing ground, providing rapid experimental confirmations $[4,5]$ for predicted radiative corrections $[6,7]$. This close interplay with theory, at the edge of experimentally accessible precision, remains the driving force behind $\pi_{e 2}$ measurements today.

\footnotetext{
${ }^{1} \mathrm{~A} \gamma$ in parentheses denotes an undetected, usually soft photon. For brevity, in further text the $(\gamma)$ will be dropped and implied; a detected photon in radiative processes will be explicitly denoted with a $\gamma$.
} 
In terms of its underlying physics, the $\pi_{e 2}$ decay stands out. By comparison, the much rarer $\mathcal{O}\left(10^{-8}\right)$ pion beta, $\pi^{+} \rightarrow \pi^{0} e^{+} v$, or $\pi_{e 3}$, decay is fully allowed in the SM. It is suppressed only by the small available final-state phase space: a major hindrance to experimental study of an otherwise ultra clean SM process. On the other hand, the strong helicity suppression of the $\pi_{e 2}$ decay (squared lepton mass ratio in Eq. (25.1)) makes this decay uniquely sensitive to a class of pseudoscalar ${ }^{2}(\boldsymbol{P})$, or $\boldsymbol{P}$-loop-coupled, non- $(V-A)$ contributions, arising from new, "beyond Standard Model" (BSM) physics, undetectable in analogous, helicity-unsuppressed leptonic decays, such as the $\pi \rightarrow \mu \bar{\nu}$, or $\pi_{\mu 2}$.

A more complete treatment of $\pi_{e 2}$ includes $\delta R_{e / \mu}^{\pi}$, the radiative and loop corrections, and the possibility of lepton universality (LU) violation, i.e., that $\boldsymbol{g}_{\boldsymbol{e}}$ and $\boldsymbol{g}_{\boldsymbol{\mu}}$, the electron and muon $W$ couplings, may not be equal:

$$
R_{e / \mu}^{\pi} \equiv \frac{\Gamma(\pi \rightarrow e \bar{\nu}(\gamma))}{\Gamma(\pi \rightarrow \mu \bar{\nu}(\gamma))}=\frac{g_{e}^{2}}{g_{\mu}^{2}} \frac{m_{e}^{2}}{m_{\mu}^{2}} \frac{\left(m_{\pi}^{2}-m_{e}^{2}\right)^{2}}{\left(m_{\pi}^{2}-m_{\mu}^{2}\right)^{2}}\left(1+\delta R_{e / \mu}^{\pi}\right) .
$$

Steady improvements of the SM description of the $\pi_{e 2}$ decay have reached the precision level of 8 parts in $10^{5}: R_{e / \mu}^{\pi, S M}=1.2352(1) \times 10^{-4}$ [8-10], which indicates that the radiative and loop corrections amount to $\sim 4 \%$ of $\boldsymbol{R}_{e / \mu}^{\pi}$. The best current experimental result, $R_{e / \mu}^{\pi, \exp }=1.2327(23) \times 10^{-4}$, dominated by measurements at TRIUMF and PSI [11-14], is 23 times less precise than the theoretical one.

The primary motivation for the PEN [15] experiment is the unique sensitivity of the $\pi_{e 2}$ decay to BSM processes is. The international PEN collaboration, led by the University of Virginia (UVa) group, set out to measure $R_{e / \mu}^{\pi}$ at PSI, with a relative precision of $\Delta R_{e / \mu}^{\pi} / R_{e / \mu}^{\pi} \leq 5 \times 10^{-4}$. At $\Delta R / R=10^{-3}, \pi_{e 2}$ probes the pseudoscalar and axial vector mass scales up to $1,000 \mathrm{TeV}$ and $20 \mathrm{TeV}$, respectively $[16,17]$. For comparison, unitarity tests of the Cabibbo-KobayashiMaskawa matrix and precise measurements of superallowed nuclear beta decays constrain the non-SM vector contributions to $>20 \mathrm{TeV}$, and scalar ones to $>10 \mathrm{TeV}$ [14]. Although scalar interactions do not directly contribute to $\boldsymbol{R}_{\boldsymbol{e} / \mu}^{\pi}$, they can do so through loop diagrams, resulting in a sensitivity to new scalar interactions up to $60 \mathrm{TeV}[16,17]$. The subject was recently reviewed in Refs. $[18,19]$. In addition, $\boldsymbol{R}_{e / \mu}^{\pi, \exp }$ provides limits on the masses of certain SUSY partners [20], and on anomalies in the neutrino sector [21]. Mounting indications [22] of $\mathrm{LU}$ violation in $\boldsymbol{B}$-meson decays make the subject additionally interesting (for a review see, e.g., [23]).

Additional goals of PEN include measurements of the radiative $\pi_{e 2 \gamma}$, and $\mu^{+} \rightarrow e^{+} \nu \bar{v} \gamma$ decays, as well as of $\tau_{\pi^{+}}$, the pion mean life. The physics motivation for the study of $\pi_{e 2 \gamma}$ decay is discussed in [24], in the context of the PiBeta experiment, predecessor to PEN. Muon decays, sensitive to non- $(V-A)$ contributions, are not discussed here; neither is $\tau_{\pi^{+}}$.

\subsection{The PEN apparatus}

As the successor to the PiBeta experiment, PEN took over the major components of this apparatus [24,25], with enhancements and upgrades. PEN detected and analyzed decays of pions and muons at rest in this detector. The 240-element pure CsI crystal calorimeter and the two multiwire proportional chambers (MWPC1,2) were serviced but otherwise unmodified. The $3.2 \mathrm{~mm}$ thick plastic hodoscope (PH) array, exhibiting surface crazing, was rebuilt with new fast $4 \mathrm{~mm}$ thick plastic scintillator staves. The central beam detectors were reconfigured, as seen in Figure 25.1.

\footnotetext{
${ }^{2}$ The $\pi^{-} \rightarrow \ell \bar{v}_{\ell}$ decay connects the pion pseudoscalar $\mathbf{0}^{-}$state to the $\mathbf{0}^{+}$vacuum.
} 


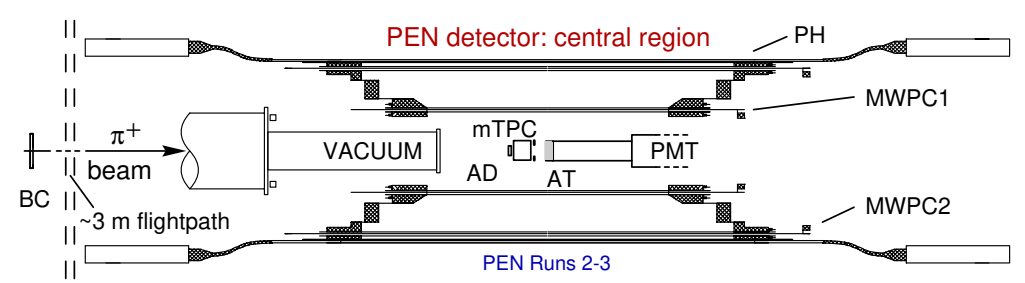

BC: upstream Beam Counter AD: Active Degrader detector AT: Active Target detector mTPC: mini Time Projection Chamber MWPC1,2: Multi-Wire Prop. Chambers $\mathrm{PH}$ : Plastic Hodoscope, 20 staves (MWPCs, PH have cylindical shape)

Figure 25.1: Schematic drawing of the central detector region in the PEN Runs 2-3 configuration, including the MTPC for beam tracking (see text for further details).

The upstream beam counter (BC) was rebuilt, the beam vacuum pipe was extended closer to the redesigned active degrader (AD) and the new one-piece active target (AT); all were optimized for the lower beam momentum $p_{\pi} \simeq 75 \mathrm{MeV} / \mathbf{c}^{2}$, compared to $\simeq 114 \mathrm{MeV} / \mathbf{c}^{2}$ in PiBeta running. The addition of PEN beam tracking detectors, discussed below, was also important. Three data-acquisition runs were completed from 2008 through 2010 in the PSI $\pi \mathrm{E} 1$ beam area. Over $\sim 25$ weeks of beam, PEN accumulated $N_{\pi \rightarrow e v} \simeq 2.3 \times 10^{7}$, and $N_{\pi \rightarrow \mu \rightarrow e}>1.5 \times 10^{8}$ events, along with significant numbers of pion and muon radiative decays.

During PEN Run 1, a plastic scintillator degrader made of four slanted wedges, was used for beam tracking. The wedge tracker was replaced in Runs 2 and 3 with low-mass mini time projection chambers, mTPCs, which improved the spatial resolution. The mTPCs, their design, performance, and the key input they provide in the analysis, are described in [26]. Signals from the beam detectors were sent to waveform digitizers, running at $2 \mathrm{GS} / \mathrm{s}$ for $\mathrm{BC}, \mathrm{AD}$, and $\mathrm{AT}$, and at $250 \mathrm{MS} / \mathrm{s}$ for the mTPC. Given the critical role of the mTPCs in controlling the $\boldsymbol{R}_{e / \mu}^{\pi}$ systematics, the analysis reported here excludes Run 1 , or $\sim 20 \%$ of the full PEN data set.

\subsection{Pion electronic decay: $\pi^{+} \rightarrow e^{+} \nu_{e}(\gamma)$}

A long list of physical processes challenge any precise measurement of $\boldsymbol{R}_{\boldsymbol{e} / \boldsymbol{\mu}}^{\pi}$ at rest, each complicating the prime objective to accurately identify, sort, count, and normalize the recorded $\pi_{e 2}$ and $\pi_{\mu 2}$ decay events. It is a particular challenge to accurately separate and count the
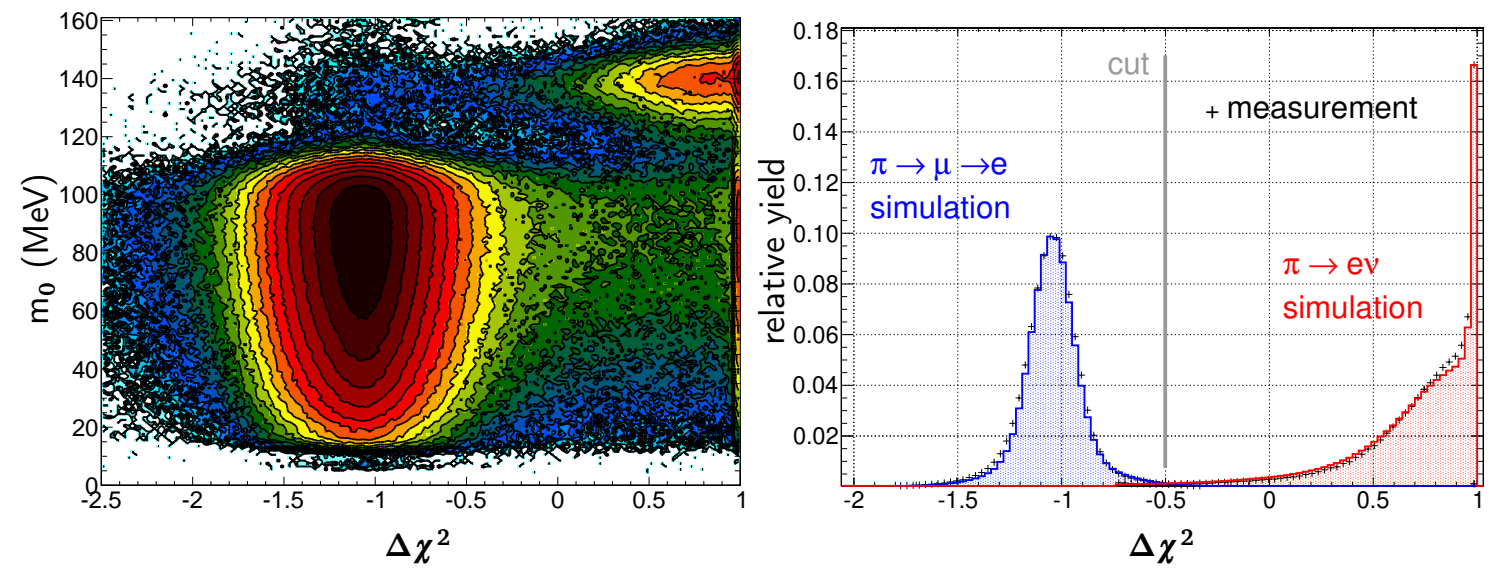

Figure 25.2: Separation of $\pi_{e 2}$ and $\pi_{\mu 2}$ events through use of the $\Delta \chi^{2}$ observable. Left: event invariant mass, $\boldsymbol{m}_{0}^{e v / e v \gamma}=\sum E_{i}+\left|\sum \vec{p}_{i}\right|$, where $i$ denotes distinct tracks/showers and $c \equiv 1$, vs. $\Delta \chi^{2}$, for a set of Run 2 data recorded with a dedicated $\pi_{\mu 2}$-suppressed trigger. Right: $\Delta \chi^{2}$ distribution for a set of standard-trigger Run 2 events. Gray line: typical choice for the cut separating the two decay types. 

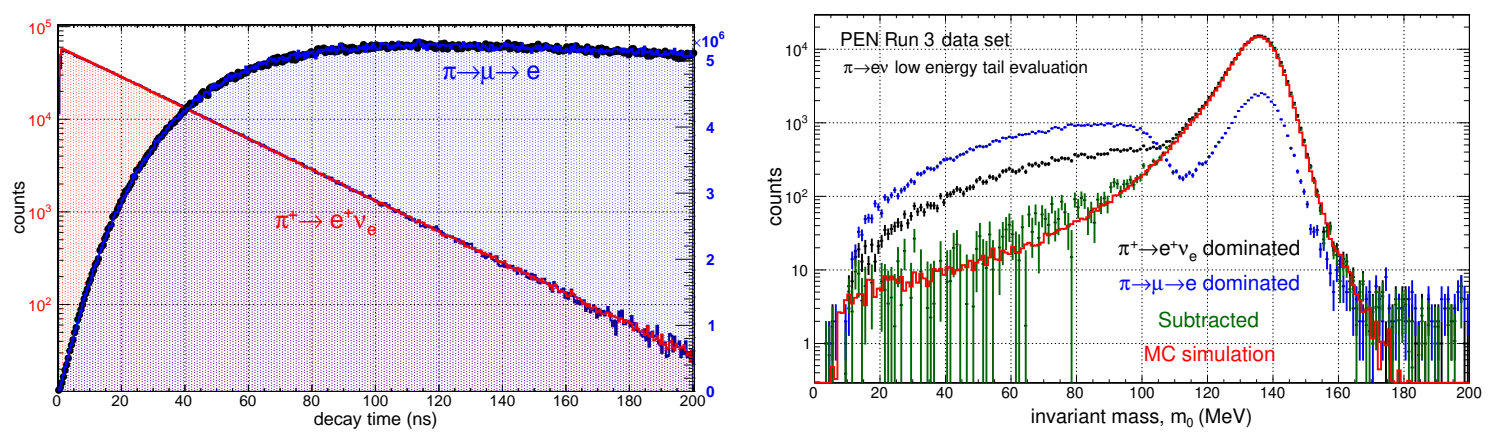

Figure 25.3: Left: decay time spectra for a subset of PEN Run 3 data (black crosses) with applied $\Delta \chi^{2}$ cuts shown in Figure 25.2. Geant4 simulation results are overlaid in red, for $\pi_{e 2}$, and blue, for $\pi_{\mu 2}$ decays. Right: low energy tail of the $\pi_{e 2}$ invariant mass, $\boldsymbol{m}_{\mathbf{0}}$, response before and after background subtraction for a subset of Run 3 data.

$\pi_{e 2}$ events that fall in the "tail" of the calorimeter energy response, under the vastly more numerous $\pi \rightarrow \mu \rightarrow e$ events. A number of observables are used to discriminate to some degree between the $\pi_{e 2}$ and $\pi_{\mu 2}$ decay events in the data. The most effective is " $\Delta \chi^{2}$ " which tests a filtered AT waveform [19] for agreement with 2-peak $\left(\pi_{e 2}\right)$, and 3-peak $\left(\pi_{\mu 2}\right)$ hypotheses based on predicted $\pi^{+}$and $e^{+}$signals (Figure 25.2).

Key to the effectiveness of the $\Delta \chi^{2}$ test are the (a) precise prediction of the decay vertex, based on the beam $\pi^{+}$and decay $\boldsymbol{e}^{+}$tracking information, plus BC-AD time of flight, and (b) accurate calibration of the AT waveform $[19,26]$.

The effectiveness of the $\Delta \chi^{2}$ discriminator is highlighted in Figure 25.3, which shows the separation of the $\pi_{e 2}$, and $\pi_{\mu 2}$ decays in the data. After subtraction of backgrounds, the best experimental determination of the low- $E$ response "tail" for $\pi_{e 2}$ events falls short of the required precision, leaving the determination of the final factor of 5 in precision (Table 25.1) to Monte Carlo simulations. This goal was recently made possible with the inclusion of corrected photoneutron cross sections $(\gamma, n)$ and $(\gamma, 2 n)$ on ${ }^{133}$ Cs and ${ }^{127} \mathrm{I}[26,27]$ into Geant4.

The experimental branching ratio $\boldsymbol{R}_{\boldsymbol{e} / \boldsymbol{\mu}}^{\pi, \exp }$ is determined as

$$
\begin{aligned}
R_{e / \mu}^{\pi, \text { exp }} & =\frac{N_{\pi \rightarrow e \nu}^{\text {peak }}\left(1+\epsilon_{\text {tail }}\right)}{N_{\pi \rightarrow \mu \nu}} \cdot \frac{f_{\pi \rightarrow \mu \rightarrow \mathrm{e}}\left(T_{\mathrm{e}}\right)}{f_{\pi \rightarrow \mathrm{e} \nu}\left(T_{\mathrm{e}}\right)} \cdot \frac{\epsilon\left(E_{\mu \rightarrow e v \bar{\nu}}\right)_{\mathrm{MWPC}}}{\epsilon\left(E_{\pi \rightarrow e \nu}\right)_{\mathrm{MWPC}}} \cdot \frac{A_{\pi \rightarrow \mu \rightarrow e}}{A_{\pi \rightarrow e \nu}} \cdot \frac{\epsilon_{\text {pileup }}}{\epsilon_{\delta \chi^{2}}} \\
& =\frac{N_{\pi \rightarrow e \nu}^{\mathrm{peak}}}{N_{\pi \rightarrow \mu \nu}} \cdot\left(1+\epsilon_{\mathrm{tail}}\right) \cdot r_{f} \cdot r_{\mathrm{PC}} \cdot r_{A} \cdot r_{\text {cut }},
\end{aligned}
$$

where $\epsilon_{\text {tail }}$ is the low energy tail fraction of the $\pi_{e 2}$ response, $r_{f}$ is the ratio of the decay fractions for the two processes within the observed decay time gates, $\boldsymbol{r}_{\mathrm{PC}}$ is the ratio of the MWPC efficiencies for the two processes, and $\boldsymbol{r}_{A}$ is the ratio of the geometrical acceptances for the two processes, evaluated from simulation. The quantities needed to determine $R_{e / \mu}^{\pi}$, given in (25.4), along with their uncertainties, are summarized in Table 25.1. As of this writing, a final critical pass through the calibration and analysis parameters is underway, so that some of the entries in the table may be improved.

\subsection{Pion radiative electronic decay: $\pi^{+} \rightarrow e^{+} \nu_{e} \gamma$}

The motivation for the measurement of the pion radiative electronic decay, $\pi_{e 2 \gamma}$, and results obtained for this channel by the PiBeta collaboration are discussed in detail in $[19,24,28]$. 
Thanks to a more open trigger, new PEN data greatly extend the phase space coverage of the $\pi_{e 2 \gamma}$ decay compared to PiBeta, of interst for determining the poorly known [28] amplitude $S D^{-} \propto\left(F_{A}-F_{V}\right)^{2}$. The region of peak sensitivity to $S D^{-}$, shown in Figure 25.4, is fully covered in PEN for the first time. The other two panels in Figure 25.4 compare the measured data and simulation using the best $F_{A, V}$ values of [28], updated in [19]. Expectations for a major breakthrough in $S D^{-}$precision are tempered, however, by the low peak $S \boldsymbol{D}^{-}$contribution $(<10 \%)$ to the differential decay rate.

\subsection{Conclusions}

The PEN collaboration is on course to improve the experimental precision of the pion electronic decay $\pi^{+} \rightarrow e^{+} v_{e}(\gamma)$ to a relative precision of $\sim 5 \times \mathbf{1 0}^{-4}$. In parallel with the current, final round of analysis parameter tuning, the collaboration is preparing for publication a series of technical papers describing the analysis, the first of which is [26]. Once the analysis is frozen, the collaboration will unblind the main result for $\boldsymbol{R}_{e / \mu}^{\pi}$. Integral to this program are the studies of radiative pion $\left(\pi_{e 2 \gamma}\right)$ and muon decays, as well as a new determination of $\tau_{\pi^{+}}$, the charged pion mean life.

\section{Acknowledgments}

This work has been supported by the U.S. National Science Foundation, the Paul Scherrer Institute, and the Russian Foundation for Basic Research.

Table 25.1: Projected uncertainty budget for the determination of $\boldsymbol{R}_{\boldsymbol{e} / \boldsymbol{\mu}}^{\pi}$ in PEN, focusing on the dominant sources. Label "DIF" denotes decay in flight of the particle so marked.

\begin{tabular}{|c|c|c|c|}
\hline Type & Observable & Value & $\Delta R_{e / \mu}^{\pi} / R_{e / \mu}^{\pi}$ \\
\hline \multirow[t]{8}{*}{ Systematic: } & $\Delta \epsilon_{\text {tail }}:$ low- $E$ "tail" fraction* & $\simeq 0.038$ & $\left\{\begin{array}{l}\simeq 0.001^{\exp } \\
2 \times\left. 10^{-4}\right|_{\text {goal }} ^{\mathrm{MC}}\end{array}\right.$ \\
\hline & $r_{f}:$ observed decay fractions & 0.0441 & $<10^{-4}$ \\
\hline & $\boldsymbol{r}_{\mathrm{PC}}$ : ratio of MWPC efficiencies & $\simeq .99$ & $<10^{-4}$ \\
\hline & $r_{A}:$ acceptance ratio (blinded) & $\simeq 1$ & $\leq 10^{-4}$ \\
\hline & $r_{\text {cut }}:$ cut efficiency ratio & $\simeq 1.0153$ & $\leq 4 \times 10^{-4}$ \\
\hline & $N_{\pi_{\mathrm{DIF} \rightarrow \mathrm{e} v}} / N_{\pi \rightarrow \mathrm{e} v}$ & $<2 \times 10^{-3}$ & $10^{-6}-10^{-5}$ \\
\hline & $N_{\pi_{\mathrm{DIF}} \rightarrow \mu \nu} / N_{\pi \rightarrow \mu \nu}$ & $2.3 \times 10^{-3}$ & $10^{-6}-10^{-5}$ \\
\hline & $N_{\mu_{D I F} \rightarrow \mathrm{e} \nu \bar{v}} / N_{\mu \rightarrow \nu \bar{v}}$ & $1.4 \times 10^{-4}$ & $<10^{-5}$ \\
\hline Statistical: & $\Delta N_{\pi \rightarrow \mathrm{e} v} / N_{\pi \rightarrow \mathrm{e} v}$ & & $\simeq 3 \times 10^{-4}$ \\
\hline Overall & goal & & $5 \times 10^{-4}$ \\
\hline
\end{tabular}



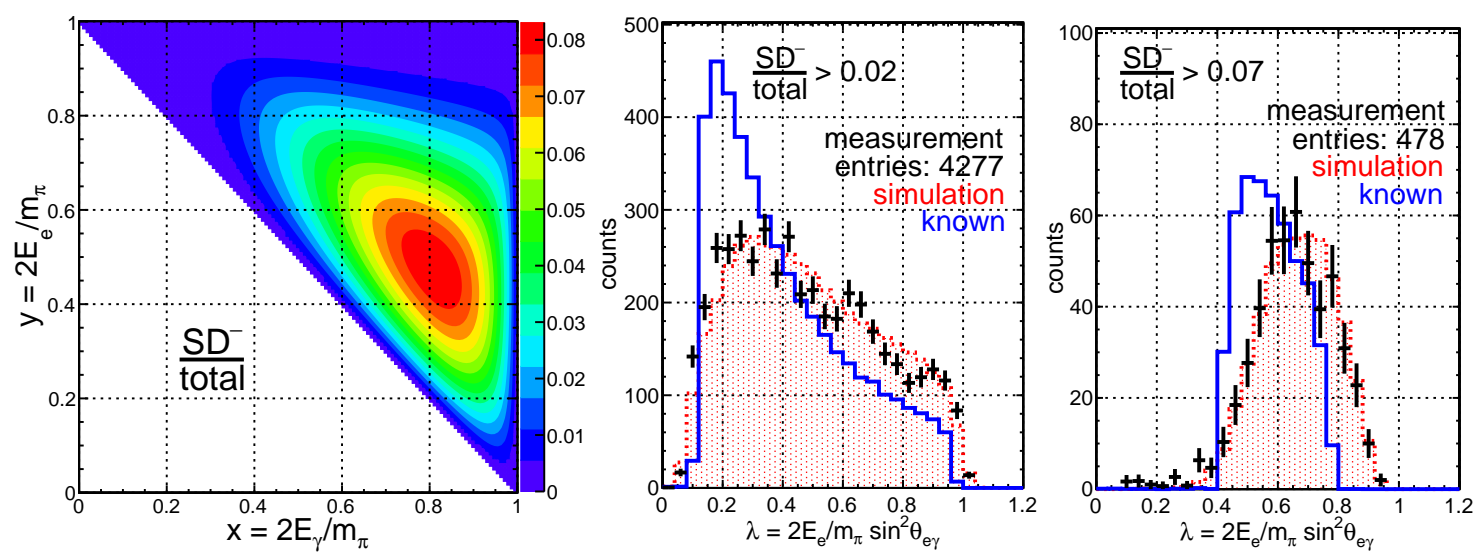

Figure 25.4: Left: phase space contours of constant fraction of the $S D^{-}$term in the overall $\pi_{e 2 \gamma}$ differential decay rate, calculated using $\boldsymbol{F}_{A, V}$ values after [28]. Center and right: measured distributions (black) in $\boldsymbol{\lambda}$ for $S D^{-} /$total $>\mathbf{0 . 0 2}$ and 0.07 , respectively, compared with Geant4 "known" values at decay event creation (blue), and results of realistic Geant4 simulation including the full effects of detector response resolution (red).

\section{References}

[1] M. Ruderman and R. Finkelstein, Note on the decay of the $\pi$-meson, Phys. Rev. 76, 1458 (1949), doi:10.1103/PhysRev.76.1458.

[2] R. P. Feynman and M. Gell-Mann, Theory of the Fermi interaction, Phys. Rev. 109, 193 (1958), doi:10.1103/PhysRev.109.193.

[3] T. Fazzini, G. Fidecaro, A. W. Merrison, H. Paul and A. V. Tollestrup, Electron decay of the pion, Phys. Rev. Lett. 1, 247 (1958), doi:10.1103/PhysRevLett.1.247.

[4] H. L. Anderson, T. Fujii, R. H. Miller and L. Tau, Branching ratio of the electronic mode of positive pion decay, Phys. Rev. 119, 2050 (1960), doi:10.1103/PhysRev.119.2050.

[5] E. Di Capua, R. Garland, L. Pondrom and A. Strelzoff, Study of the decay $\pi \rightarrow \boldsymbol{e} \boldsymbol{v}$, Phys. Rev. 133, B1333 (1964), doi:10.1103/PhysRev.133.B1333.

[6] S. M. Berman, Radiative corrections to pion beta decay, Phys. Rev. Lett. 1, 468 (1958), doi:10.1103/PhysRevLett.1.468.

[7] T. Kinoshita, Radiative corrections to $\pi$-e decay, Phys. Rev. Lett. 2, 477 (1959), doi:10.1103/PhysRevLett.2.477.

[8] W. J. Marciano and A. Sirlin, Radiative corrections to $\pi_{l 2}$ decays, Phys. Rev. Lett. 71, 3629 (1993), doi:10.1103/PhysRevLett.71.3629.

[9] M. Finkemeier, Radiative corrections to $\pi_{l 2}$ and $\boldsymbol{K}_{l 2}$ decays, Phys. Lett. B 387, 391 (1996), doi:10.1016/0370-2693(96)01030-1.

[10] V. Cirigliano and I. Rosell, Two-loop effective theory analysis of $\pi(K) \rightarrow e \bar{v}_{e}(\gamma)$ branching ratios, Phys. Rev. Lett. 99, 231801 (2007), doi:10.1103/PhysRevLett.99.231801.

[11] D. I. Britton et al., Measurement of the pi+ $\rightarrow e+$ neutrino branching ratio, Phys. Rev. Lett. 68, 3000 (1992), doi:10.1103/PhysRevLett.68.3000. 
[12] G. Czapek et al., Branching ratio for the rare pion decay into positron and neutrino, Phys. Rev. Lett. 70, 17 (1993), doi:10.1103/PhysRevLett.70.17.

[13] A. Aguilar-Arevalo et al., Improved measurement of the $\pi \rightarrow e v$ branching ratio, Phys. Rev. Lett. 115, 071801 (2015), doi:10.1103/PhysRevLett.115.071801.

[14] P. Zyla et al., Review of particle physics, Progr. Theor. Exper. Phys. $083 C 01$ (2020), doi:10.1093/ptep/ptaa104.

[15] Pen experiment home page, http://pen.phys.virginia.edu.

[16] B. A. Campbell and D. W. Maybury, Constraints on scalar couplings from, Nucl. Phys. B 709, 419 (2005), doi:10.1016/j.nuclphysb.2004.12.015.

[17] B. A. Campbell and A. Ismail, Leptonic pion decay and physics beyond the electroweak Standard Model (2008), arXiv:0810.4918.

[18] D. A. Bryman, P. Depommier and C. Leroy, $\pi \rightarrow \boldsymbol{e} \boldsymbol{\nu}, \pi \rightarrow \boldsymbol{e} \boldsymbol{\nu \gamma}$ decays and related processes, Phys. Rep. 88, 151 (1982), doi:10.1016/0370-1573(82)90162-4.

[19] D. Počanić, E. Frlež and A. van der Schaaf, Experimental study of rare charged pion decays, J. Phys. G: Nucl. Part. Phys. 41, 114002 (2014), doi:10.1088/09543899/41/11/114002.

[20] M. J. Ramsey-Musolf, S. Su and S. Tulin, Pion leptonic decays and supersymmetry, Phys. Rev. D 76, 095017 (2007), doi:10.1103/PhysRevD.76.095017.

[21] W. Loinaz, N. Okamura, S. Rayyan, T. Takeuchi and L. C. R. Wijewardhana, NuTeV anomaly, lepton universality, and nonuniversal neutrino-gauge couplings, Phys. Rev. D 70, 113004 (2004), doi:10.1103/PhysRevD.70.113004.

[22] L. C. R. Aaij et al., Test of lepton universality in beauty-quark decays (2021), arXiv:2103.11769.

[23] G. Ciezarek, M. Franco Sevilla, B. Hamilton, R. Kowalewski, T. Kuhr, V. Lüth and Y. Sato, A challenge to lepton universality in B-meson decays, Nature 546, 227 (2017), doi:10.1038/nature22346.

[24] D. Počanić, The pion beta and radiative electronic decays, SciPost Phys. Proc. 5, 024 (2021), doi:10.21468/SciPostPhysProc.5.024.

[25] E. Frlež et al., Design, commissioning and performance of the PIBETA detector at PSI, Nucl. Instrum. Meth. A526, 300 (2004), doi:10.1016/j.nima.2004.03.137.

[26] C. J. Glaser, D. Pocanic, A. van der Schaaf, V. A. Baranov, N. V. Khomutov, N. P. Kravchuk and N. A. Kuchinsky, Beam particle tracking with a low-mass mini time projection chamber in the PEN experiment (2020), arXiv:2012.12266.

[27] C. Glaser, D. Počanić and V. Baranov, Monte Carlo simulation of the $(\boldsymbol{\gamma}, \boldsymbol{n})$ and $(\boldsymbol{\gamma}, \mathbf{2 n})$ reactions on cesium and iodine below $100 \mathrm{MeV}$, in preparation (2021).

[28] M. Bychkov et al., New precise measurement of the pion weak form factors in $\pi^{+} \rightarrow e^{+} \boldsymbol{v \gamma}$ decay, Phys. Rev. Lett. 103, 051802 (2009), doi:10.1103/PhysRevLett.103.051802. 